\title{
Diagnosing radio plasma heating in the polar summer mesosphere using cross modulation: Theory and observations
}

\author{
A. Senior, ${ }^{1,2}$ M. T. Rietveld, ${ }^{3}$ M. J. Kosch,,${ }^{1,2}$ and W. Singer ${ }^{4}$ \\ Received 19 February 2010; revised 28 April 2010; accepted 18 May 2010; published 21 September 2010.
}

[1] Radio heating of the free electrons in the mesosphere is an important diagnostic technique for the phenomenon of polar mesosphere summer echoes (PMSE). Due to a lack of suitable observations, the heating must be modeled when studying its effect on PMSE. In order to try to validate these models, the cross-modulation technique was implemented at the EISCAT facility near Tromsø, Norway to compare the absorption of a diagnostic radio wave in the heated and unheated plasma. The results are compared to predictions from a model similar to that used for estimating the heating in PMSE studies. It is found that, after allowing for certain instrumental effects, the model overestimates the change in absorption of the diagnostic wave by a factor of 1.5-2.5. It is suggested that the assumption that the electron distribution function remains Maxwellian during heating could contribute to this discrepancy.

Citation: Senior, A., M. T. Rietveld, M. J. Kosch, and W. Singer (2010), Diagnosing radio plasma heating in the polar summer mesosphere using cross modulation: Theory and observations, J. Geophys. Res., 115, A09318, doi:10.1029/2010JA015379.

\section{Introduction}

[2] In recent years there has been considerable interest in the phenomenon of polar mesospheric summer echoes (PMSE), strong radar returns from the vicinity of the mesopause at high latitudes during the summer. PMSE are thought to be caused by the charging of minute (tens of nanometer) ice particles by the free electrons in the $\mathrm{D}$ region ionosphere which occupies the same altitude region. The charged ice particles lead to irregular electron density structures that can backscatter radio waves. Similar ice particles are also thought to be responsible for polar mesospheric or noctilucent clouds. A recent review of PMSE has been given by Rapp and Lübken [2004].

[3] A powerful ground-based diagnostic for PMSE is the use of high-power HF radio waves to heat the electron gas. This increases the diffusion rate of the electrons, smoothing out the irregular structure and partially suppressing the PMSE [Rapp and Lübken, 2000]. The characteristics of the temporal response of PMSE to electron heating can in principle allow the determination of the amount and size of the dust particles [Havnes et al., 2003; Havnes, 2004]. However, in the work on radio wave heating of PMSE to date, the electron temperature increase due to the radio wave has been calculated theoretically [e.g., Belova et al., 2001;

\footnotetext{
${ }^{1}$ Department of Communication Systems, Lancaster University, Lancaster, UK.

${ }^{2}$ Also at Department of Physics, Lancaster University, Lancaster, UK.

${ }^{3}$ EISCAT Scientific Association, Ramfjordbotn, Norway.

${ }^{4}$ Leibniz-Institute of Atmospheric Physics, Rostock University, Kühlungsborn, Germany.

Copyright 2010 by the American Geophysical Union. 0148-0227/10/2010JA015379
}

Havnes et al., 2007], due to the difficulty of measuring it in this region.

[4] Whilst incoherent scatter radar is capable of determining the electron temperature in the $\mathrm{E}$ and $\mathrm{F}$ regions [Evans, 1969], the interpretation of the backscatter spectrum from the D region is complicated. Recently, some progress has been made on this using new techniques [Kero et al., 2008], but it is desirable to have another method with which to compare these results.

[5] The technique of ionospheric cross modulation, reviewed by Fejer [1970], relies on the modified absorption experienced by other radio waves passing through a region of heated electrons due to the increased electron-neutral collision frequency there. A cross-modulation experiment involves two transmitters. In the pulsed cross-modulation method introduced by Fejer [1955], one transmitter radiates a pulsed low-power wave which is usually reflected from the ionospheric $\mathrm{E}$ region. A second, higher-power transmitter radiates another pulsed wave which may or may not be reflected from the ionosphere. Its purpose is to disturb the $\mathrm{D}$ region by causing electron heating and modifying the absorption of the low-power wave. The modification of the absorption is detected by comparing the strength of the ionospheric echo of the low-power wave received back on the ground with that obtained when the high-power transmitter is switched off. In cross-modulation terminology, the low-power wave is usually referred to as the "wanted" wave and the high-power wave as the "disturbing" wave. By arranging the disturbing pulse to follow the wanted pulse, the disturbing pulse intersects the downcoming wanted pulse at a height dependent on the delay between the pulses, Figure 1. This gives the experiment some ability to distinguish the heating effect of the disturbing wave as a function of height. 


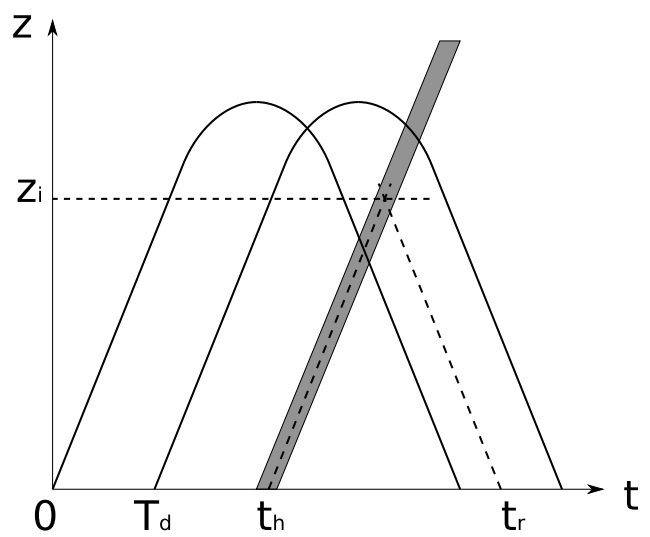

Figure 1. Range-time diagram of the diagnostic and disturbing pulses (shaded) as they propagate through the ionosphere. The diagnostic pulse begins at $t=0$ and ends at $t=T_{d}$. The disturbing pulse of length $T_{h}$ is emitted centered at $t=t_{h}$ after alternate diagnostic pulses. The diagnostic echo received at time $t_{r}$ corresponds to the intersection of the diagnostic and disturbing pulses at altitude $z_{i}=c\left(t_{r}-t_{h}\right) / 2$, the "interaction height". This diagram neglects any ionospheric group delay of the disturbing pulse.

[6] The absorption coefficient of a radio wave in a plasma basically depends upon the wave frequency, the electron density and the electron collision frequency [Hargreaves, 1992]. Since the wave frequency is known and if the electron density can be measured, the electron collision frequency can be determined and from this, the electron temperature can be deduced using empirical formulae [Schunk and Nagy, 1978]. The experiment described herein had this as its original goal. However, as described in the remainder of the text, a considerable discrepancy was found between the modeled and observed cross modulation, with the model overestimating the observed effect. Sulzer et al. [1982] performed a similar study to the present one, but using the high-power $430 \mathrm{MHz}$ Arecibo incoherent scatter radar as the disturbing transmitter rather than a high-power HF transmitter. They also found that their model of the heating overestimated the observations.

\section{Instrumentation}

[7] The diagnostic (wanted) wave was generated by the EISCAT Dynasonde ionospheric sounder, located at Ramfjordmoen near Tromsø, Norway $\left(69.58^{\circ} \mathrm{N}, 19.22^{\circ} \mathrm{E}\right)$ [Rietveld et al., 2008]. The transmitter radiated a $200 \mu \mathrm{s}$ pulse every $10 \mathrm{~ms}$ for the first $48 \mathrm{~s}$ of each UT minute, with a ERP (effective radiated power relative to an isotropic radiator) of $\sim 1200 \mathrm{~W}$ on a frequency of $2 \mathrm{MHz}$.

[8] The disturbing wave was generated by the EISCAT HF facility, colocated with the Dynasonde [Rietveld et al., 1993]. The RF-on pulse from the Dynasonde was fed to a circuit which generated a secondary pulse with an adjustable delay after every other diagnostic pulse. This secondary pulse was used to modulate the disturbing wave. The secondary pulse was $50 \mu$ s long and delayed to start at the end of the Dynasonde pulse. The delay was set manually by comparing the RF pulse envelopes on an oscilloscope. We estimate that an error of $\pm 10 \mu \mathrm{s}$ in the delay is quite likely as the pulse edges are not sharply defined. This leads to an uncertainty of $\pm 1.5 \mathrm{~km}$ in the interaction altitude (defined in section 4). The HF facility was configured to radiate vertically with $\mathrm{X}$ mode polarization on $5.423 \mathrm{MHz}$. The ERP was varied as described later. Figure 1 shows a range-time diagram of the diagnostic and disturbing pulses as they propagate through the ionosphere.

[9] The ionospherically reflected diagnostic pulse was received by the Dynasonde using a pair of orthogonal dipole antennae connected to two separate receiver channels. The receiver mixed the $2 \mathrm{MHz}$ signal to quadrature baseband form and recorded it to disk with $10 \mu \mathrm{s}$ sampling. The receiver bandwidth was $30 \mathrm{kHz}$ and the impulse response was $\sim 50 \mu$ s long, leading to a range resolution of $\sim 7.5 \mathrm{~km}$.

[10] During the experiment, the EISCAT VHF radar [Rishbeth and van Eyken, 1993] at the same location measured the electron densities vertically overhead between altitudes of 60 and $140 \mathrm{~km}$ with a resolution of $300 \mathrm{~m}$ using the arc dlayer program. The measured densities below $\sim 90 \mathrm{~km}$ were low and somewhat unreliable due to the poor signal-to-noise ratio. Additional electron density measurements from the MF radar at Saura, Andøya $\left(69.3^{\circ} \mathrm{N}, 16.0^{\circ} \mathrm{E}\right)$, approximately $130 \mathrm{~km}$ WSW of Ramfjordmoen were also used in this study. The MF radar is described by Singer et al. [2008].

\section{Cross-Modulation Data Analysis}

[11] As described in section 2, the Dynasonde recorded data in 1 min blocks containing the measured echo voltages from the first $48 \mathrm{~s}$ of the minute. As the interpulse period was $10 \mathrm{~ms}$, each block contains 4800 samples at each of 154 range gates spanning the interval of virtual ranges 69 $298.5 \mathrm{~km}$ in $1.5 \mathrm{~km}(10 \mu \mathrm{s})$ steps. There are two such sets of samples, one for each receiver/antenna, which we denote $z_{1, j}$ and $z_{2, j}$ where $j=1,2, \ldots 4800$ is the pulse number.

[12] The first step of processing subtracts any d.c. offset from the samples and scales them to allow for the receiver attenuator setting. The samples from the two receiver channels are then summed with the second channel phaseshifted by $\pm 90^{\circ}$ to form sample vectors for the $\mathrm{O}$ and $\mathrm{X}$ mode components of the echo

$$
\begin{gathered}
z_{\mathrm{O}, j}=z_{1, j}-i z_{2, j} \\
z_{\mathrm{X}, j}=z_{1, j}+i z_{2, j} .
\end{gathered}
$$

[13] The $\mathrm{O}$ or $\mathrm{X}$ mode samples are then divided into two sets formed from alternate pulses. This separates the diagnostic pulses which are or are not followed by a disturbing pulse

$$
\begin{gathered}
z_{\mathrm{on}, k}=z_{\mathrm{O} / \mathrm{X}, 2 k-1} \\
z_{\mathrm{off}, k}=z_{\mathrm{O} / \mathrm{X}, 2 k}
\end{gathered}
$$

where $k=1,2, \ldots 2400$ and the subscripts "on" and "off" refer to the diagnostic pulses which are and are not followed by a disturbing pulse. 
[14] The instantaneous echo power is then computed as

$$
P_{\text {on } / \text { off }, k}=\left|z_{\text {on } / \text { off }, k}\right|^{2} .
$$

[15] The mean background noise power $N_{\text {on/off } k}$ in a set of range gates where no echo is present during the sounding is then subtracted from the echo power. Denoting the background-subtracted power as $P^{\prime}=P-N$, the crossmodulation power ratio $m$ is then calculated simply as

$$
m=\sum_{k=1}^{k=2400} P_{\mathrm{off}, k}^{\prime} / \sum_{k=1}^{k=2400} P_{\mathrm{on}, k}^{\prime} .
$$

This is then converted into decibels to give the "diagnostic absorption change"

$$
\Delta A=10 \log _{10} m
$$

which is positive when the absorption of the diagnostic is increased by the disturbing pulse $(m>1)$.

\section{Results}

[16] Here, we describe the results from the experiment performed on 2 July 2008. The experiment was divided into two parts. The first part ("Case 1") ran from 10:55 to 11:20 UT and the second part ("Case 2") from 11:32 to 12:00 UT. During Case 1, the HF facility used the high-gain antenna array (Array 1) with a ERP of $630 \mathrm{MW}$ and a halfpower beam width of $7.3^{\circ}$. During Case 2 , the low-gain array (Array 2) was used giving a ERP of $228 \mathrm{MW}$ and a beam width of $9.6^{\circ}$.

[17] Figure 2 summarizes the results of the 2 July 2008 experiment. The top frame shows the vertical electron density profile measured by the VHF radar as a function of time. The rapid increase in density above $\sim 90 \mathrm{~km}$ is easily seen. The thin layer of enhanced electron density around $85 \mathrm{~km}$ is in fact a PMSE layer, not a true density enhancement. Another thin layer beginning at $100 \mathrm{~km}$ and descending and weakening during the run is a sporadic-E layer. The vertical stripes which appear from time to time are caused by echoes from meteor trails or spacecraft. The line plot shows that the density at $90 \mathrm{~km}$ increases during Case 1 then remains more or less steady.

[18] The second frame presents the results of analyzing the cross-modulation experiment itself. The profile of change in absorption of the diagnostic pulse versus the "interaction altitude" is shown as a function of time. The "interaction altitude" is defined as the altitude at which the center of the upgoing disturbing pulse intersects the downcoming diagnostic pulse, see Figure 1. The altitude is calculated assuming straight line propagation at the free space speed of light. Since the group velocity of the diagnostic wave falls below this as it approaches reflection, the true altitude is overestimated close to the reflection height. Only the $\mathrm{O}$ mode echo is presented because the X mode echo was too weak to be of use. It is seen that the diagnostic absorption change is very small below $\sim 70 \mathrm{~km}$ and then rises to $\sim 0.3 \mathrm{~dB}$ in the $70-90 \mathrm{~km}$ interval. The positive change in absorption indicates that the disturbing wave enhances the absorption of the diagnostic in this altitude interval. It appears from both the color and line plots that the diagnostic absorption change is more variable in Case 1 than in Case 2.

[19] The third frame shows the echo power of the diagnostic wave as a function of time and virtual range (the apparent range assuming propagation at the free space speed of light). The $30 \mathrm{~km}$ range extent of the $200 \mu$ s pulse can be seen, with the echo centered a little below $100 \mathrm{~km}$. The true height of reflection, determined from the electron density profiles was $\sim 94 \mathrm{~km}$. As explained in the previous paragraph, the group delay of the pulse as it approaches reflection leads to the apparent detection of cross modulation above this true height of reflection. Referring to the line plot, the echo power declines somewhat during Case 1 then fluctuates during Case 2. The background noise is about $5 \mathrm{~dB}$ higher in Case 2 than in Case 1.

\section{Model}

\subsection{Time-Dependent Heating Model}

[20] In order to interpret the results, a model of the radio wave heating of the plasma and the absorption of the diagnostic wave was constructed. The model is similar to previous ones used for modeling heating of PMSE [Belova et al., 1995; Kassa et al., 2005] and in the interpretation of incoherent scatter observations during $\mathrm{D}$ region heating [Kero et al., 2007]. It differs from these because the short time scales involved in the cross-modulation experiment require modeling of the time-dependent heating, not just the equilibrium state. Time-dependent models for cross modulation were used by, for example, Fejer [1955] and Weisbrod et al. [1964]. The present model includes the selfabsorption of the high-power HF heating wave which was neglected in these models, probably due to the lower powers used. The numerical model described by Georges [1966] does include this effect but it is not clear whether the model used by Sulzer et al. [1982] did so. The present model also has some refinements in the radio absorption calculation and in the use of more recent electron cooling rates.

[21] The model divides the $\mathrm{D}$ region into a series of contiguous layers in altitude. Each layer has a thickness of $300 \mathrm{~m}$ as determined by the VHF radar data. Starting with the lowest layer at altitude $z_{0}$, the power flux of the disturbing wave into the layer is described by

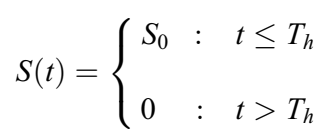

where $S_{0}=\mathrm{ERP} / 4 \pi z_{0}^{2}$ is the power flux during the disturbing pulse of length $T_{h}$. This power flux function is then used to solve the time-dependent electron energy equation

$$
\frac{3}{2} k_{B} N_{e} \frac{\mathrm{d} T_{e}}{\mathrm{~d} t}=-2 S(t) \frac{\omega}{c} \operatorname{Im} \mu\left(N_{e}, T_{e}\right)-N_{e} L\left(T_{e}, T_{n}\right)
$$

where $k_{B}$ is Boltzmann's constant, $N_{e}, T_{e}$ are the electron number density and temperature, $\omega$ is the disturbing wave angular frequency, $c$ is the free space speed of light, $\mu$ is the complex refractive index of the disturbing wave and $L\left(T_{e}, T_{n}\right)$ is the electron energy loss function where $T_{n}$ is the 


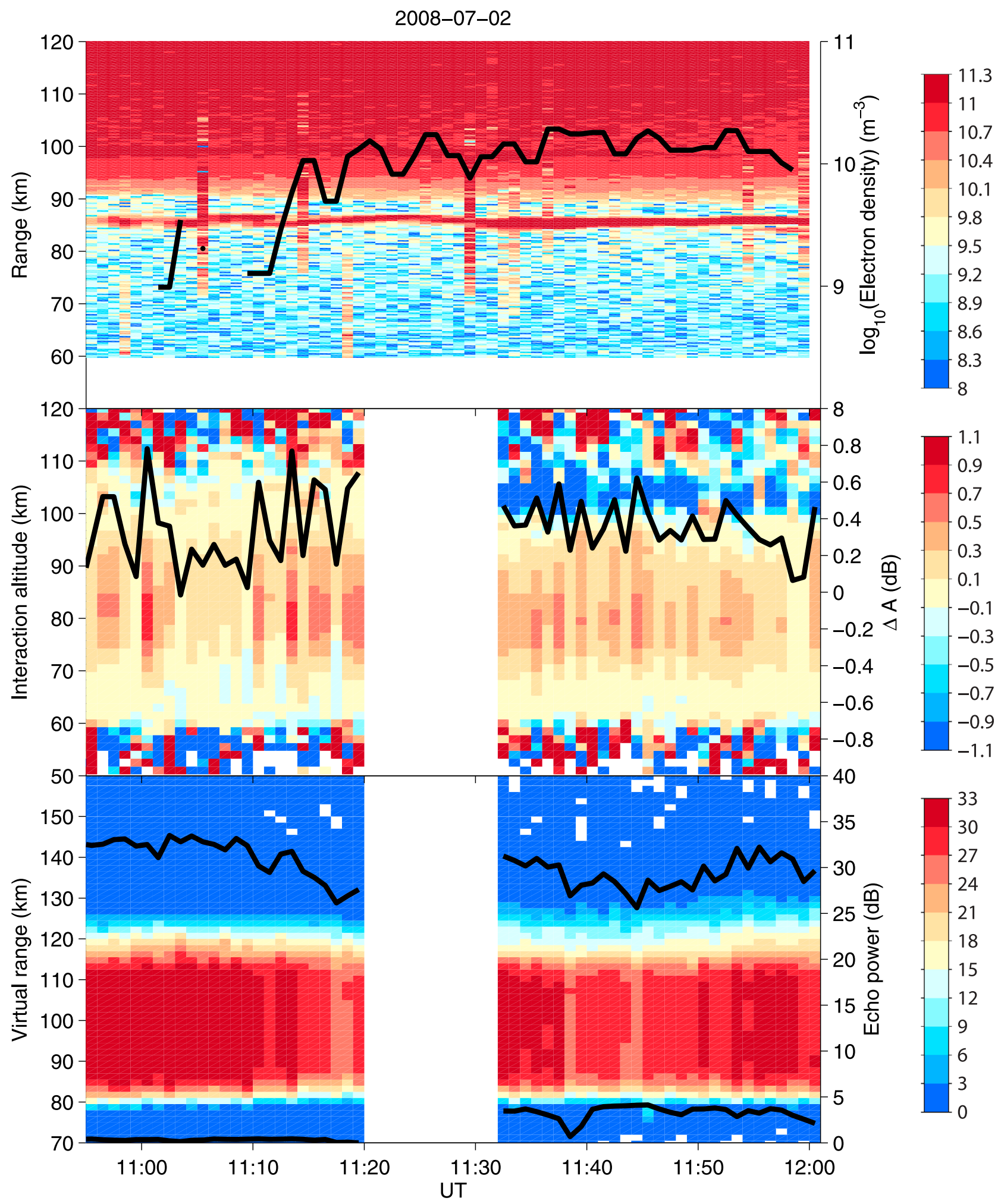

Figure 2. Observations from 2 July 2008. (top) Electron density from the EISCAT VHF radar versus altitude and time. The black line shows the density at $90 \mathrm{~km}$, filtered with a three-point median filter in time. (middle) The change in absorption of the diagnostic wave from the undisturbed to the disturbed ionosphere versus interaction altitude and time. The black line shows the absorption change at $80 \mathrm{~km}$. (bottom) The power of the diagnostic echo versus virtual range and time. The upper black line is the power at $100 \mathrm{~km}$. The lower black line is the background noise power. Refer to the right-hand scales in each frame for the lines. 


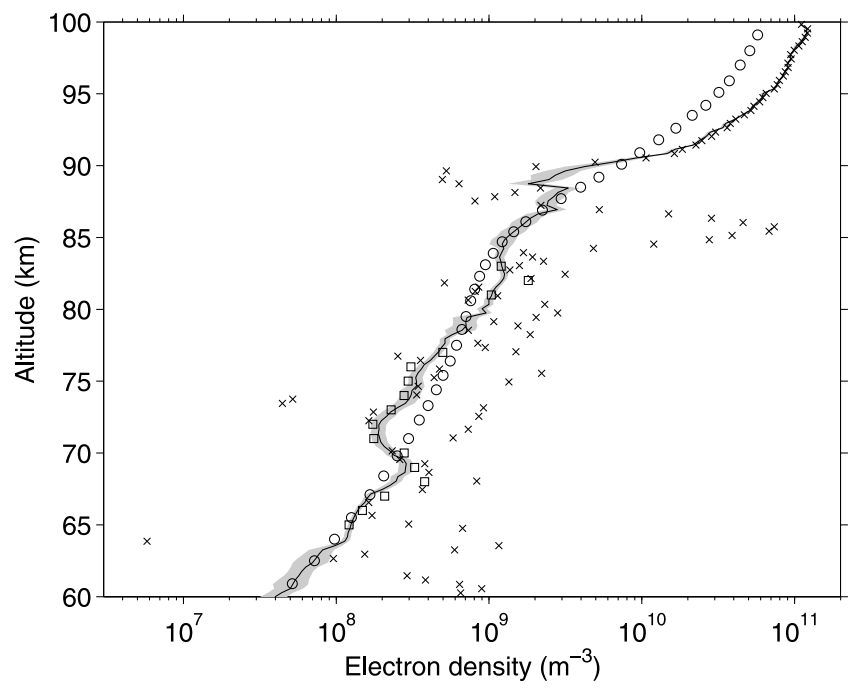

Figure 3. The electron density profile (black line) used for modeling in Case 1. The gray band denotes the one standard error bound on the profile. The crosses are measurements from the EISCAT VHF radar, the squares are measurements from the Saura MF radar, and the circles are estimates from the IMAZ model. The error estimates for the measurements/ model are not shown as the plot would be too cluttered.

neutral gas temperature. The first term on the right represents the energy gained by electrons due to the absorption of the disturbing wave.

[22] The complex refractive index $\mu$ comes from the formula of Garrett [1985] which allows the velocity dependence of the electron-neutral collision frequency to be arbitrary; essentially a further generalization of the expression of Sen and Wyller [1960]. In this case, the collision frequencies are calculated as a function of electron energy from the electron-neutral momentum transfer cross sections. For $\mathrm{N}_{2}$ these cross sections come from Morrison et al. [1997] for energies below $0.01 \mathrm{eV}$ and from Tabata et al. [2006] above $0.01 \mathrm{eV}$; for $\mathrm{O}_{2}$ they come from Lawton and Phelps [1978] for energies below $1 \mathrm{eV}$ and from Sullivan et al. [1995] for energies above $1 \mathrm{eV}$. Collisions with $\mathrm{O}$ are neglected since the concentration of $\mathrm{O}$ is negligible over most of the altitude range of interest.

[23] The electron energy loss function $L\left(T_{e}, T_{n}\right)$ represents cooling of the electrons by rotational and vibrational excitation of $\mathrm{N}_{2}$ and $\mathrm{O}_{2}$. For $\mathrm{N}_{2}$, the expressions of Pavlov [1998a] are used. For $\mathrm{O}_{2}$, the expression of Pavlov [1998b] is used for rotational excitation and the expression of Jones et al. [2003] is used for vibrational excitation.

[24] Having solved (9) for $T_{e}(t)$, the power flux of the disturbing wave leaving the layer having been reduced by beam divergence and absorption is calculated as

$$
S_{\text {out }}(t)=\left(\frac{z}{z+\delta z}\right)^{2} S(t)+2 S(t) \delta z \frac{\omega}{c} \operatorname{Im} \mu\left(N_{e}, T_{e}\right)
$$

where $\delta z$ is the thickness of the layer. The first term represents beam divergence, the second absorption. This new power flux function $S_{\text {out }}(t)$ then forms the input power flux to the layer above the current one and the calculation proceeds as for the previous layer until the uppermost layer has been calculated. A time shift of $\delta z / c$ is applied between each layer to represent the propagation of the disturbing wave. The concentrations of the neutral species $\mathrm{N}_{2}, \mathrm{O}_{2}$ and $\mathrm{O}$ and the neutral temperature $T_{n}$ are taken from the MSISE90 model [Hedin, 1991].

[25] At the end of this process, there is a model representation of the heating due to the disturbing wave as a function of altitude and time. The model then calculates the absorption of the diagnostic wave through this time-varying medium and calculates the difference between this absorption and that in the undisturbed ionosphere as a function of time. In doing so, altitudes where the group velocity of the wave falls below $0.9 c$ as the wave approaches reflection are neglected and the change in absorption taken as zero. This avoids the need for a full ray-tracing solution to accurately determine the absorption of the diagnostic wave, but is only valid because reflection of the diagnostic occurs in a region where the disturbance of the ionosphere due to heating is small.

[26] Finally, the absorption change is integrated along the path of the downcoming diagnostic wave to give the diagnostic absorption change $\Delta A$ due to the disturbing wave which can be compared to that observed.

\subsection{Electron Density Profiles for Modeling}

[27] As mentioned in section 2, the electron densities measured by the VHF radar below $\sim 90 \mathrm{~km}$ have a high variance due to the low signal-to-noise ratio. In order to construct a profile over an altitude interval sufficient to interpret the observations $(60-100 \mathrm{~km})$, we used two other sources of information. Firstly, the measured densities from the MF radar at Saura and secondly, modeled densities from the IMAZ empirical model [McKinnell and Friedrich, 2006].

[28] The median of the VHF radar density profiles within the time interval of interest (Case 1 or 2) was taken, $N_{e, \mathrm{VHF}}$ $(z)$. The median was chosen to reduce the influence of profiles contaminated by meteor or spacecraft echoes. The variance of the median, $\sigma_{\mathrm{VHF}}^{2}(z)$ was estimated by a bootstrap approach. The mean Saura MF radar density profile $N_{e}$, $\mathrm{MF}_{2}(z)$ was taken over each interval along with its variance $\sigma_{\mathrm{MF}}^{2}$ determined from the individual profiles from both the differential absorption and differential phase determinations of the density from the partial reflection measurements. Altitudes at which fewer than three measurements were available in each interval were neglected. The IMAZ model was run to give another density profile $N_{e, \text { IMAZ }}$. The model itself provides the necessary variance $\sigma_{\mathrm{IMAZ}}^{2}$.

[29] The profiles were then merged to form a single profile $N_{e}(z)$ by weighting them according to their variances

$$
N_{e}=\frac{N_{e, \mathrm{VHF}} / \sigma_{\mathrm{VHF}}^{2}+N_{e, \mathrm{MF}} / \sigma_{\mathrm{MF}}^{2}+N_{e, \mathrm{IMAZ}} / \sigma_{\mathrm{IMAZ}}^{2}}{1 / \sigma_{\mathrm{VHF}}^{2}+1 / \sigma_{\mathrm{MF}}^{2}+1 / \sigma_{\mathrm{IMAZ}}^{2}} .
$$

[30] The Saura measurements were only available in a limited altitude interval and outside this interval, the variance $\sigma_{\mathrm{MF}}^{2}$ was effectively taken as infinite.

[31] Figure 3 shows the resulting profile for Case 1 together with the individual measurements and model values used to construct it. Below $65 \mathrm{~km}$, the profile follows the IMAZ model (circles). Between 65 and $84 \mathrm{~km}$ it is influ- 

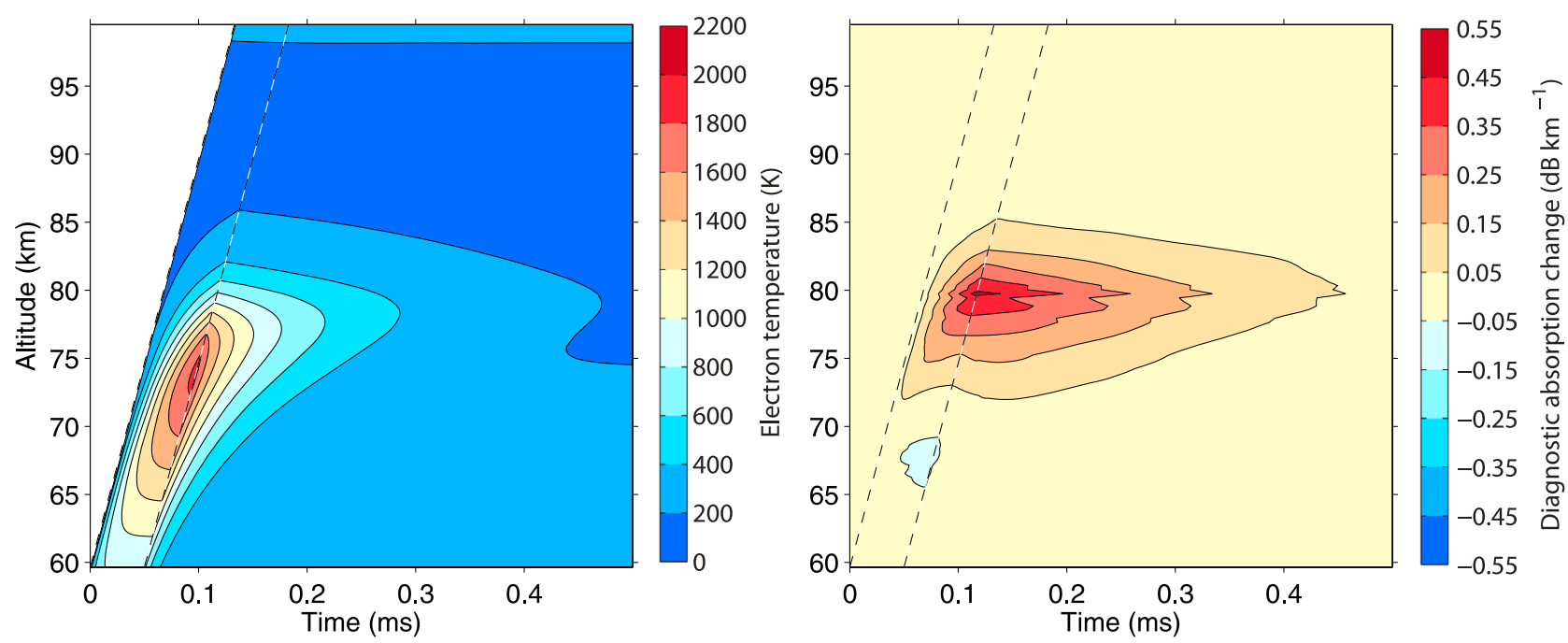

Figure 4. Heating model results for Case 1. (left) The electron temperature versus altitude and time. (right) The change in diagnostic absorption per unit path length from the unheated to the heated ionosphere. The dashed lines show the propagation of the disturbing pulse.

enced by both the model and the Saura MF radar data (squares). Between 84 and $87 \mathrm{~km}$ the profile follows the model again and above $87 \mathrm{~km}$ the profile follows the EISCAT VHF measurements. The large peak in the VHF measurements around $85 \mathrm{~km}$ is due to the PMSE layer and is not true electron density. These points have large variances and therefore do not greatly influence the final profile. Below about $70 \mathrm{~km}$, the VHF measurements appear to tend toward a constant density around $5 \times 10^{8} \mathrm{~m}^{-3}$. This is an artifact of the logarithmic scale. At these altitudes, the noise level of the VHF data exceeds the electron density and many density values are negative; these cannot be represented on the logarithmic scale.

\subsection{Model Results}

[32] Figure 4 shows an example of the model output for Case 1. The left frame of Figure 4 is the electron temperature as a function of altitude and time. The increase in heating and cooling time scales with altitude is clearly seen. The peak temperature occurs between 70 and $75 \mathrm{~km}$. The right frame shows the change in absorption per unit path length experienced by the diagnostic wave propagating through the heated ionosphere shown on the left compared to the unheated ionosphere. Notice that at lower altitudes (below about $70 \mathrm{~km}$ ), the absorption is decreased by heating whereas at higher altitudes it is increased. The peak increase occurs just between 75 and $80 \mathrm{~km}$.

[33] Figure 5 compares the observations and model results for both cases. In Case 1 (left) there is considerable variability between individual $60 \mathrm{~s}$ soundings, but taking into account the different absorption scales, the modeled absorption change overestimates that observed by about a factor of 10. The shape of the observed and modeled profiles also seems quite different with the modeled profile peaking at $85 \mathrm{~km}$ whereas the observed profiles peak at $83 \mathrm{~km}$ or lower.

[34] The right frame of Figure 5 shows the same comparison for Case 2. Here the model overestimates the observations by a factor of 5. Again, the model profile peaks higher in altitude than the observed profiles.

\section{Discussion}

[35] Figure 5 shows that there is a considerable difference between the observed and modeled diagnostic absorption changes. The difference, although still a factor of 5, is also a factor of 2 less in Case 2 than in Case 1. The observed diagnostic absorption changes also seem to be more variable in Case 1 than in Case 2. The difference between the cases, apart from any changes in the ionosphere, is that in Case 2, the disturbing transmitter used a wider beam $\left(9.6^{\circ}\right.$ rather than $7.3^{\circ}$ ) and a correspondingly lower ERP (228 MW rather than $630 \mathrm{MW}$ ).

[36] It was noted in section 2 that there was a $\pm 1.5 \mathrm{~km}$ uncertainty in the measured interaction altitude and that the range resolution was $\sim 7.5 \mathrm{~km}$. Although displacing the observed profiles in Figure 5 in altitude relative to the model results would improve the agreement in the shape of the profile, it would not improve the agreement in magnitude. In addition, the modeled profiles are sufficiently slowly varying in altitude that smoothing them to take account of the range resolution would not greatly alter their magnitude.

\subsection{Effective Field of View of the Diagnostic}

[37] First, a possible source of systematic error in the cross-modulation measurement is explored. It is well known [Ratcliffe, 1957] that the ionosphere is not a smooth reflecting surface and that the reflection of a vertically incident wave consists of contributions from a "patch" of ionosphere rather than a single point. The Dynasonde has large transmitting and receiving beam widths (approximately $102^{\circ}$ and $90^{\circ}$, respectively) so it is possible that diagnostic echoes arriving from angles off the zenith traverse a region of ionosphere that is less strongly affected by the heating, diluting the cross-modulation effect. Indeed, it is noted in section 3 of Fejer [1970] that an experiment using a narrow transmitting beam "was too directional for 

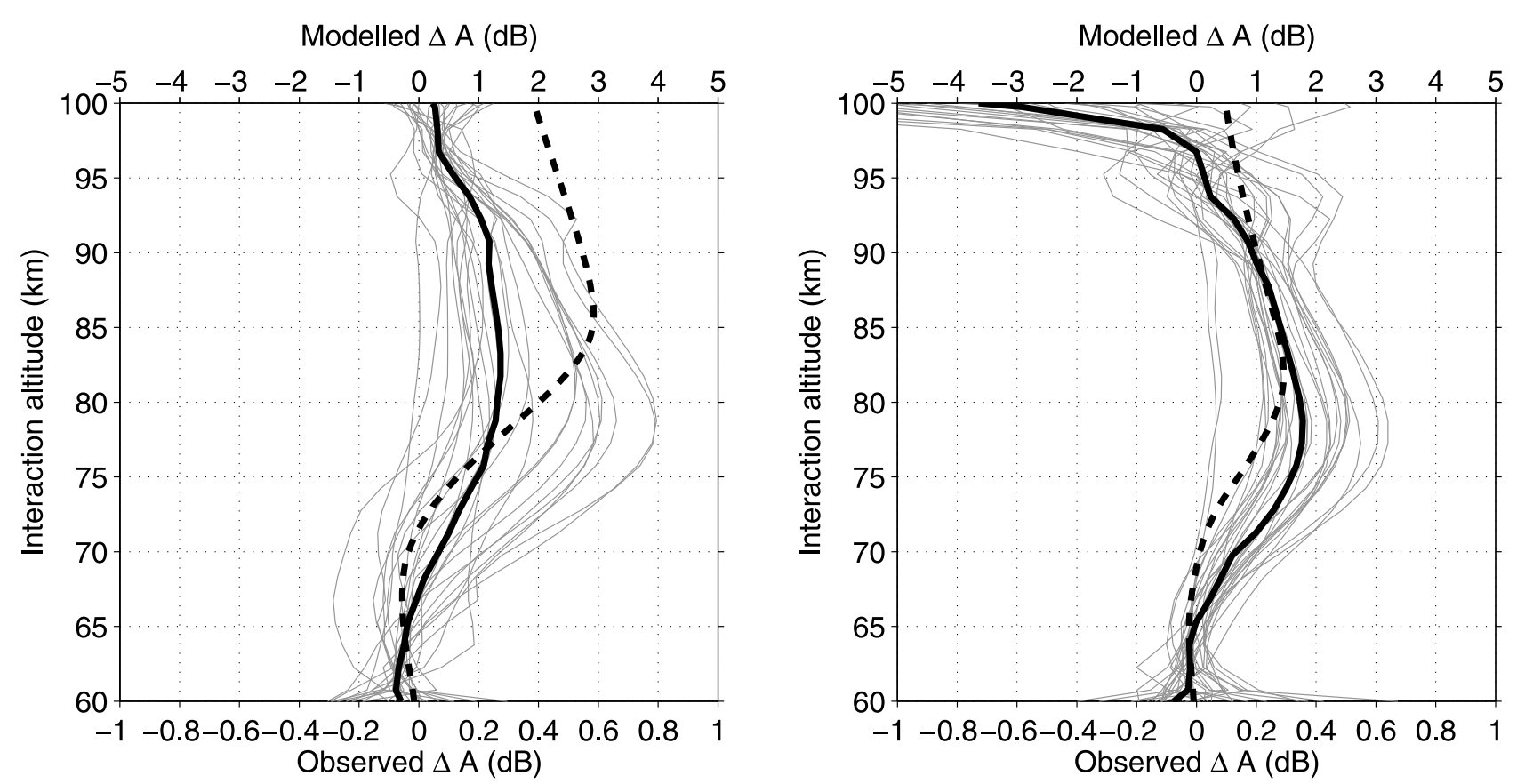

Figure 5. Comparison of observations and modeling for (left) Case 1 and (right) Case 2. The thin gray lines are the diagnostic absorption change profiles for each $60 \mathrm{~s}$ sounding. The heavy black line is the median of these profiles; refer to the lower scale. The heavy dashed line is the modeled diagnostic absorption change profile; refer to the upper scale.

pulsed cross-modulation measurements because the fading wanted echo arrives from a cone whose opening angle usually exceeds the width of the transmitted beam". Sulzer et al. [1982] had a particularly severe example of this effect because their heater beam was the very narrow $430 \mathrm{MHz}$ Arecibo radar beam.

[38] In order to simulate this, the model of section 5.1 was extended to three dimensions in space as described in Appendix A. In this model, the power of the diagnostic wave reflected from the ionosphere is assumed to be a Gaussian function of zenith angle. Changing the half-angle of this Gaussian changes the diluting effect of off-zenith contributions to the received signal. The purely vertical propagation case studied so far corresponds to a half-angle approaching zero. Sulzer et al. [1982] adopted another approach based on a Fresnel zone theory of the reflected diagnostic wave. Figure 6 is similar to Figure 5 but compares the model results for a set of half-angles for Cases 1 and 2 to the corresponding observations. It is clear that increasing the half-angle does indeed reduce the modeled diagnostic absorption change as expected.

[39] Unfortunately, it is not possible to determine the halfangle of the reflecting region in the case of the 2 July 2008 experiment. However, on 4 December 2009 between 08:16 and 08:22 UT, a series of measurements were made with the EISCAT Dynasonde, using the spaced receiving antennae to measure the half-angle by interferometry. The result was a half-angle of the angular power spectrum of $\sim 5^{\circ}$ for frequencies of 1.9 and $2.4 \mathrm{MHz}$. This is within the range of values quoted by Ratcliffe [1957] for similar frequencies. It can be seen from Figure 6 that at such a half-angle, the discrepancy between theory and observation is reduced to a factor of about 2.5 in Case 1 and about 1.5 in Case 2. However, it is clear that no single half-width gives good agreement for both cases.

\subsection{Model Errors}

[40] Turning attention to errors in the modeling, in section 5.2 the estimation of the electron density profile was described. From Figure 3 it is evident that in the $80-85 \mathrm{~km}$ interval where the VHF radar measurements have not been completely overcome by noise, the densities obtained suggest that the profile adopted for the model could be too low by a factor of about 2, although this would contradict the MF radar measurements. Also, some of the MF radar profiles (not shown) indicated the presence of an electron "biteout" [Rapp and Lübken, 2004] at the height of the PMSE layer. However, this depletion in density would probably make little difference to the model results since it occurs at too high an altitude (see Figures 2 and 4). In order to gauge what effect uncertainty in the electron density might have on the model results, the model was rerun with the whole electron density profile scaled by factors of 2 and 0.5 , for the purely zenithal propagation case. When the densities are scaled by a factor of 2 , the peak $\Delta A$ for Case 1 (Case 2) becomes $3.8 \mathrm{~dB}(1.8 \mathrm{~dB})$ compared to $2.9 \mathrm{~dB}(1.5 \mathrm{~dB})$ before scaling. For a scaling by 0.5 , the corresponding peak $\Delta A$ is $1.9 \mathrm{~dB}(0.9 \mathrm{~dB})$. This indicates that the model results are relatively robust to uncertainty in the electron density.

[41] Another source of error in the model could be the neutral gas densities which are required for calculating the collision frequencies (part of the refractive index calculation) and the electron cooling rates. These come from the MSISE-90 model [Hedin, 1991]. However, Hedin [1991] 

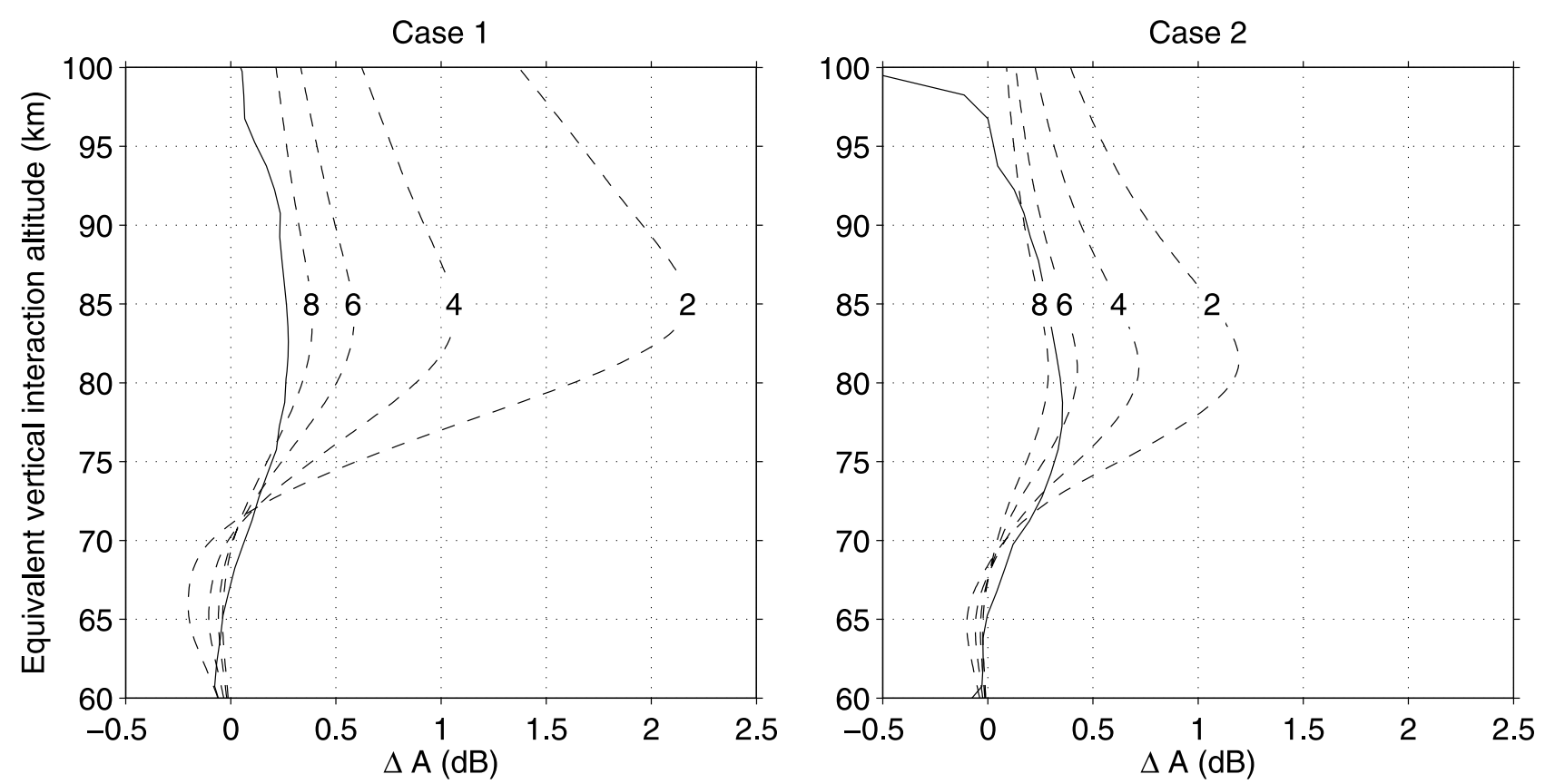

Figure 6. (left) Comparison of observed median diagnostic absorption change (solid line) for Case 1 with model results for source half-widths of $2^{\circ}, 4^{\circ}, 6^{\circ}$, and $8^{\circ}$ (dashed lines with half-widths indicated). (right) As for Figure 6 (left), but for Case 2.

indicates that the model densities fit those observed to within about $5 \%$ in the mesosphere. More recently, Beharrell and Honary [2008] used data from EISCAT to test the accuracy of collision frequencies estimated from the NRLMSISE-00 model [Picone et al., 2002], which is similar to MSISE-90 in the mesosphere and found no evidence of a serious discrepancy. Lübken and von Zahn [1991] and Lübken [1999] summarize data from a variety of measurements made at $69-70^{\circ} \mathrm{N}$. The neutral mass densities from these results agree with the MSISE-90 mass densities used here to within $3 \%$ between 70 and $90 \mathrm{~km}$ altitude and within $10 \%$ between 60 and $70 \mathrm{~km}$ altitude. Hence, it seems unlikely that an error in the neutral densities could give rise to the large difference between theory and observation found here.

[42] The calculation of the heat source and absorption of the diagnostic wave in the model are both based on a kinetic theory [Garrett, 1985], but this makes the assumption that the electron distribution function remains Maxwellian during the heating. Similarly, the electron cooling rates are derived from cross-section data under the assumption of a Maxwellian electron distribution [Pavlov, 1998a, 1998b; Jones et al., 2003]. It was shown theoretically by Stubbe [1981] that there can in fact be significant departures from a Maxwellian distribution. Unfortunately, this theory is only for steady state conditions and is therefore not directly applicable to cross modulation. Garrett [1991] derived a kinetic theory of cross modulation, but this theory does not include all of the inelastic collision processes identified by Stubbe [1981] as being important. The application of these theories is beyond the scope of the present work, but could play a role in resolving the remaining discrepancy between theory and observation.

\subsection{Relation to Previous Results}

[43] Sulzer et al. [1982] performed a similar experiment to the one described herein, the main difference being the use of the $430 \mathrm{MHz}$ Arecibo radar as the heating transmitter which led to a particularly severe difference between the size of the heated region and the field of view of their diagnostic sounder. They found that their model of the cross modulation also overestimated that observed and suggested that the electron cooling rates used could be too small by a factor of about 4. However, their modeling did not make use of electron densities measured during the actual conditions of the experiment as in the present results and the cooling rates in question were drawn from older sources [Schunk and Nagy, 1978; Banks and Kockarts, 1973]. The total cooling rate due to vibrational and rotational excitation of $\mathrm{O}_{2}$ and $\mathrm{N}_{2}$ used in the present work is approximately $75 \%$ of that obtained from the expressions in the work of Schunk and Nagy [1978] for $200 \leq T_{e} \leq 2000 \mathrm{~K}$ and then rises to be approximately $175 \%$ of it for $T_{e} \geq 3000 \mathrm{~K}\left(T_{n}=200 \mathrm{~K}\right.$ was assumed). Hence, it seems likely that had the older cooling rates been used in the present study, the modeled electron temperatures would have been lower and the agreement with the observations improved. On the other hand, had Sulzer et al. [1982] used the more recent cooling rates, they would have found an even larger discrepancy between theory and observation. Sulzer et al. [1982] also included cooling due to fine structure excitation of $\mathrm{O}$, but the concentration of this species is very small over most of the height interval of interest.

[44] The recent incoherent scatter measurements of $\mathrm{D}$ region electron heating by the EISCAT HF facility described by Kero et al. [2008] agreed well with a similar type of model to that used here in two of the three cases 
considered. In the third case, the model overestimated the observed heating, though the authors suggested that this could have been due to contamination of the observations by polar mesospheric winter echoes (PMWE). On the other hand, Kero et al. [2007] found that the model overestimated the change in cosmic noise absorption at $38.2 \mathrm{MHz}$ due to radio electron heating from EISCAT by a factor of 5-10. The modulation of cosmic noise absorption is similar to cross modulation, with the cosmic radio noise playing the role of the diagnostic wave but at a much higher frequency that the $2 \mathrm{MHz}$ diagnostic used here. Kero et al. [2007] suggested that their refractive index model [Sen and Wyller, 1960] might be invalid in the highly collisional $\mathrm{D}$ region or that the electron cooling rates were heavily underestimated.

[45] Finally, it is worth bearing in mind that the measurements reported and analyzed here are predominantly in nonequilibrium heating conditions. For example, it is clear from the model calculations in Figure 4 that at the heights from which the greatest contribution to the diagnostic absorption change comes (around $80 \mathrm{~km}$ ), the electron temperature has not reached equilibrium in the duration of the heating pulse. On the other hand, the observations reported by Kero et al. [2007] involve much longer heating times for which equilibrium would have been reached. This is also the case in the type of PMSE modulation experiments discussed by Havnes et al. [2003]. It is conceivable that the discrepancy between theory and observation found here might be different in equilibrium conditions, depending on the reason for this discrepancy.

\subsection{Future Experiments}

[46] If the type of experiment described here was to be repeated in the future, a useful improvement would be the use of the interferometric capability of the Dynasonde to determine the angular spread of the reflected diagnostic wave while the experiment is in progress. This would allow the correct half-angle to be used in the modeling. The heater beam could also be steered in zenith angle to explore the contribution of off-zenith reflection of the diagnostic wave to the received signal. Although the analysis has not been carried out with the present data set, the data would also allow the phase cross modulation [Weisbrod et al., 1964] to be calculated and used in addition to the power cross modulation used here.

\section{Conclusion}

[47] The results of a cross-modulation experiment using a high-power HF transmitter to disturb the ionosphere in conditions when PMSE were present have been described. Calculations using a model similar to those used to estimate the artificially enhanced electron temperature in PMSE modulation experiments overestimate the cross modulation by a factor of about 1.5-2.5, even after the effective field of view of the diagnostic sounder has been accounted for. Uncertainty in the electron density and the neutral atmosphere density seems insufficient to explain the discrepancy, though it is suggested that the assumption that the electron distribution remains Maxwellian during heating of the plasma, which is implicit in the model, is another potential source of error. The results suggest that some caution should be used when applying similar models of $\mathrm{D}$ region electron heating in quantitative studies.

\section{Appendix A: Three-Dimensional Heating Model}

[48] In order to calculate the three-dimensional spatial distribution of heating, we first of all assume axial symmetry about the vertical. The power flux from the heater can be approximated by

$$
S(\theta)=(\sin k \theta / k \theta)^{2}
$$

where $\theta$ is the zenith angle $k=2.783 / \Delta \theta$ and $\Delta \theta$ is the halfpower beam width in radians. Note that the axial symmetry is not strictly true for the square planar phased array antenna system used. This power flux model can then be used in conjunction with a version of the heating model described in section 5.1 which accounts for the increased path length due to off-vertical propagation through the ionosphere.

[49] We next assume that the Dynasonde sees the diagnostic wave arriving from a fictitious axially symmetric source in (or beyond) the ionosphere. The power flux from this source can be represented as

$$
J(\theta)=J_{0} \exp \left(-\theta^{2} / 2 \theta_{0}^{2}\right)
$$

where $\theta$ is again zenith angle and $\theta_{0}$ is the half-angle of the source region. The power flux passes through the absorbing ionosphere before reaching the receiver. The amplitude scaling factor due to absorption in a direction $\theta$ at time $t$ is given by

$$
A(\theta, t)=\exp \left[-\int \kappa(\theta, r, t) \mathrm{d} r\right]
$$

where $\kappa$ is the absorption coefficient and $r$ is range from the receiver. In the absence of heating, $\kappa=\kappa_{\text {off }}(z)$ can be assumed independent of zenith angle and time. Furthermore, since we are only interested in relatively small zenith angles $\theta$, we can neglect the distinction between range and altitude and the absorption scaling factor becomes

$$
A_{\text {off }}=\exp \left[-\int \kappa_{\text {off }}(z) \mathrm{d} z\right]
$$

whereas in the presence of heating we have

$$
A_{\text {on }}(\theta, t)=\exp \left[-\int \kappa_{\text {on }}(\theta, r, t) \mathrm{d} r\right] .
$$

[50] If we now assume that the waves from different parts of the source are incoherent (randomly phased), the power fluxes from different directions can be summed to give the total power at the receiver

$$
P(t)=2 \pi \int J(\theta) A(\theta, t)^{2} \sin \theta \mathrm{d} \theta .
$$

[51] Here we also make the assumption that the Dynasonde transmitting and receiving antenna gain is constant over the range of zenith angles of interest, which seems 
reasonable given the large beam widths. The modeled diagnostic absorption change is then given by

$$
\begin{gathered}
\Delta A=10 \log _{10} \frac{P_{\text {on }}(t)}{P_{\text {off }}} \\
=10 \log _{10} \frac{\int J(\theta)\left(A_{\text {on }}(\theta, t) / A_{\text {off }}\right)^{2} \sin \theta \mathrm{d} \theta}{\int J(\theta) \sin \theta \mathrm{d} \theta} .
\end{gathered}
$$

[52] Acknowledgments. We are grateful to O. Havnes (University of Tromsø) for supporting a collaborative visit funded by a Royal Society award to A.S. during which the experiment was performed and for his useful comments on the manuscript. We also thank M. Beharrell and B. Gustavsson (Lancaster) for helpful discussions. EISCAT is an international association supported by research organizations in China (CRIRP), Finland (SA), Germany (DFG), Japan (NIPR and STEL), Norway (NFR), Sweden (VR), and the United Kingdom (STFC). This work was also supported by STFC grant ST/F003005/1.

[53] Bob Lysak thanks the reviewers for their assistance in evaluating this paper.

\section{References}

Banks, P. M., and G. Kockarts (1973), Aeronomy, Part B, Academic, London.

Beharrell, M., and F. Honary (2008), A new method for deducing the effective collision frequency profile in the D region, J. Geophys. Res., 113, A05303, doi:10.1029/2007JA012650.

Belova, E., P. Chilson, M. Rapp, and S. Kirkwood (2001), Electron temperature dependence of PMSE power: Experimental and modelling results, Adv. Space Res., 28(7), 1077-1082.

Belova, E. G., A. B. Pashin, and W. B. Lyatsky (1995), Passage of a powerful HF radio wave through the lower ionosphere as a function of initial electron density profiles, J. Atmos. Terr. Phys., 57, 265-272.

Evans, J. V. (1969), Theory and practice of ionosphere study by Thomson scatter radar, Proc. IEEE, 57(4), 496-530.

Fejer, J. A. (1955), The interaction of pulsed radio waves in the ionosphere, J. Atmos. Terr. Phys., 7, 322-332.

Fejer, J. A. (1970), Radio wave probing of the lower ionosphere by crossmodulation techniques, J. Atmos. Terr. Phys., 32, 597-607.

Garrett, A. J. M. (1985), Multispecies kinetic generalization of the AppletonHartree dispersion formula, J. Plasma Phys., 33, 265-284.

Garrett, A. J. M. (1991), Kinetic theory of cross modulation in a weakly ionized plasma, J. Plasma Phys., 46, 365-390.

Georges, T. M. (1966), A numerical method for interpreting D region radio pulse interaction measurements, Radio Sci., 1, 1077-1088.

Hargreaves, J. K. (1992), The Solar-Terrestrial Environment, Cambridge Univ. Press, Cambridge, U. K.

Havnes, O. (2004), Polar mesospheric summer echoes (PMSE) overshoot effect due to cycling of artificial electron heating, J. Geophys. Res., 109, A02309, doi:10.1029/2003JA010159.

Havnes, O., C. La Hoz, L. I. Næsheim, and M. T. Rietveld (2003), First observations of the PMSE overshoot effect and its use for investigating the conditions in the summer mesosphere, Geophys. Res. Lett., 30(23), 2229, doi:10.1029/2003GL018429.

Havnes, O., M. Kassa, and C. LaHoz (2007), Time evolution of artificial electron heating in polar mesosphere summer echo layers, J. Geophys. Res., 112, D08202, doi:10.1029/2006JD007660.

Hedin, A. E. (1991), Extension of the MSIS thermosphere model into the middle and lower atmosphere, J. Geophys. Res., 96, 1159-1172, doi:10.1029/90JA02125.

Jones, D. B., L. Campbell, M. J. Bottema, and M. J. Brunger (2003), New electron energy transfer rates for vibrational excitation of $\mathrm{O}_{2}, \mathrm{New} J$. Phys., 5(1), 114.1-114.11.

Kassa, M., O. Havnes, and E. Belova (2005), The effect of electron biteouts on artificial electron heating and the PMSE overshoot, Ann. Geophys., 23, 3633-3643.

Kero, A., C.-F. Enell, T. Ulich, E. Turunen, M. T. Rietveld, and F. H. Honary (2007), Statistical signature of active D-region HF heating in IRIS riometer data from 1994-2004, Ann. Geophys., 25, 407-415.
Kero, A., J. Vierinen, C.-F. Enell, I. Virtanen, and E. Turunen (2008), New incoherent scatter diagnostic methods for the heated $\mathrm{D}$ region ionosphere, Ann. Geophys., 26, 2273-2279.

Lawton, S. A., and A. V. Phelps (1978), Excitation of the $b^{1} \Sigma_{g}^{+}$state of $\mathrm{O}_{2}$ by low energy electrons, J. Chem. Phys., 69, 1055-1068.

Lübken, F.-J. (1999), Thermal structure of the Arctic summer mesosphere, J. Geophys. Res., 104, 9135-9149, doi:10.1029/1999JD900076.

Lübken, F.-J., and U. von Zahn (1991), Thermal structure of the mesopause region at polar latitudes, J. Geophys. Res., 96, 20,841-20,857, doi:10.1029/91JD02018.

McKinnell, L. A., and M. Friedrich (2006), Results from a new auroral lower ionosphere model, Adv. Space Res., 37, 1045-1050, doi:10.1016/j.asr.2004.11.008.

Morrison, M. A., W. Sun, W. A. Isaacs, and W. K. Trail (1997), Ultrasimple calculation of very low energy momentum transfer and rotationalexcitation cross section: $e-\mathrm{N}_{2}$ scattering, Phys. Rev. A, 55, 2786-2798.

Pavlov, A. V. (1998a), New electron energy transfer rates for vibrational excitation of $\mathrm{N}_{2}$, Ann. Geophys., 16, 176-182.

Pavlov, A. V. (1998b), New electron energy transfer and cooling rates by excitation of $\mathrm{O}_{2}$, Ann. Geophys., 16, 1007-1013.

Picone, J. M., A. E. Hedin, D. P. Drob, and A. C. Aikin (2002), NRLMSISE-00 empirical model of the atmosphere: Statistical comparisons and scientific issues, J. Geophys. Res., 107(A12), 1468, doi:10.1029/2002JA009430.

Rapp, M., and F.-J. Lübken (2000), Electron temperature control of PMSE, Geophys. Res. Lett., 27(20), 3285-3288, doi:10.1029/2000GL011922.

Rapp, M., and F.-J. Lübken (2004), Polar mesosphere summer echoes (PMSE): Review of observations and current understanding, Atmos. Chem. Phys., 4, 2601-2633.

Ratcliffe, J. A. (1957), Some aspects of diffraction theory and their application to the ionosphere, Rep. Prog. Phys., 19, 188-267.

Rietveld, M. T., H. Kohl, H. Kopka, and P. Stubbe (1993), Introduction to ionospheric heating experiments at Tromsø: I. Experimental overview, J. Atmos. Terr. Phys., 55, 577-599.

Rietveld, M. T., J. W. Wright, N. Zabotin, and M. L. V. Pitteway (2008), The Tromsø dynasonde, Polar Sci., 2, 55-71, doi:10.1016/j. polar.2008.02.001.

Rishbeth, H., and A. P. van Eyken (1993), EISCAT—Early history and the first ten years of operation, J. Atmos. Terr. Phys., 55(4-5), 525-542.

Schunk, R. W., and A. F. Nagy (1978), Electron temperatures in the F region of the ionosphere: Theory and observations, Rev. Geophys., 16(3), 355-399.

Sen, H. K., and A. A. Wyller (1960), On the generalization of the AppletonHartree magnetoionic formulas, J. Geophys. Res., 65(12), 3931-3950, doi:10.1029/JZ065i012p03931.

Singer, W., R. Latteck, and D. A. Holdsworth (2008), A new narrow beam Doppler radar at $3 \mathrm{MHz}$ for studies of the high-latitude middle atmosphere, Adv. Space Res., 41, 1488-1494, doi:10.1016/j.asr.2007.10.006.

Stubbe, P. (1981), Modifying effects of a strong electromagnetic wave upon a weakly ionized plasma: A kinetic description, Radio Sci., 16(3), 417-425, doi:10.1029/RS016i003p00417.

Sullivan, J. P., J. C. Gibson, R. J. Gulley, and S. J. Buckman (1995), Lowenergy electron scattering from $\mathrm{O}_{2}$, J. Phys. B At. Mol. Opt. Phys., 28, 4319-4328.

Sulzer, M. P., J. D. Mathews, and A. A. Tomko (1982), A UHF crossmodulation D region heating experiment with aeronomic implications, Radio Sci., 17(2), 435-443, doi:10.1029/RS017i002p00435.

Tabata, T., T. Shirai, M. Sataka, and H. Kubo (2006), Analytic cross sections for electron impact collisions with nitrogen molecules, At. Data Nucl. Data Tables, 92, 375-406.

Weisbrod, S., A. J. Ferraro, and H. S. Lee (1964), Investigation of phase interaction as a means of studying the lower ionosphere, J. Geophys. Res., 69(11), 2337-2348, doi:10.1029/JZ069i011p02337.

M. J. Kosch and A. Senior, Department of Communication Systems, Lancaster University, South Drive, Lancaster LA1 4WA, UK. (m.kosch@ lancaster.ac.uk; a.senior@lancaster.ac.uk)

M. T. Rietveld, EISCAT Scientific Association, Ramfjordmoen, N-9027 Ramfjordbotn, Norway. (mike.rietveld@eiscat.uit.no)

W. Singer, Leibniz-Institute of Atmospheric Physics, Rostock University, Schlossstrasse 6, D-18225 Kühlungsborn, Germany. (singer@ iap-kborn.de) 